\title{
Research on Application of Railway Construction Management System Based on EA and SOA
}

\author{
ZHI Peng ${ }^{+}$, SHI Tian-yun, XIE Ya-long and CHEN Xun \\ China Academy of Railway Sciences Corporation Limited
}

\begin{abstract}
According to the railway construction reality of covering a wide scope, having many participant organizations, containing complex construction constitutions, and leading to variant business applications, a general solution of railway construction management system based on the combination of SOA and EA is proposed to solve the problems of isolation, heterogeneity, closure and integration difficulty which exist among nowaday railway construction information systems. First, the technical implementation path is given by doing research into the existing system's key technology. Second, after an intense analyze and study on the overall architecture, information architecture, application architecture, technology architecture and network security, the problems of complicated business reconstruction, data integration and service sharing in the railway construction management system have been solved. The business data resource can be maximum reuse to meet the actual needs of railway construction business management. Last, combined with the verification of construction application, the top view of architecture allows a variety of elements to be examined and optimized in a unified framework. both the construction safety and quality can be guaranteed, maximizing the teamwork efficiency and reducing the construction costs.
\end{abstract}

Keywords: information technology, railway construction project, EA, SOA, railway construction management system, enterprise service bus.

\section{Introduction}

Railway construction projects involve a wide range, many participating units, complex construction organizations, and diverse business applications. Existing railway construction informatization systems have problems such as loose management and coordination mechanisms, independent closure of business systems, and difficulties in data isolation and integration, which seriously restricts the safety and efficiency of railway construction. In order to achieve data integration, application sharing, and business collaboration in railway construction management systems, it is necessary to realize system integration and business process integration through functional modularization and servitization. This requires that the overall architecture of the system is flexible and expandable, and on the basis of supporting componentized and integrated system functions, it must also meet the requirement of the reorganization and rearrangement of business processes.

With the development of IT technology, EA (Enterprise Architecture) and SOA (Service Oriented Architecture) are gradually merged to form a new architecture theory.

EA refers to the general solution provided to solve the systematic and universal issues in the enterprise information management system. It is based on business oriented and driven architecture to understand, analyze, design, build, integrate, expand, operate and manage information systems. According to the Federal Enterprise Architecture Framework (FEAF), the enterprise architecture consists of two main components, the business architecture and the IT architecture (as shown in Figure 1).

SOA is a distributed component model, which is implemented through functional modularization and servitization. It can be used to implement distributed deployment, combination, and use of loosely coupled

${ }^{+}$Corresponding author. Tel.: +86-18600416111; fax: +86-01051849176.

E-mail address: zprails@qq.com. 
coarse-grained application components through the network according to actual application requirements [1] (The SOA architecture is shown in Figure 2). It has features such as coarse granularity, loose coupling, location transparency, protocol independence, and shared service.

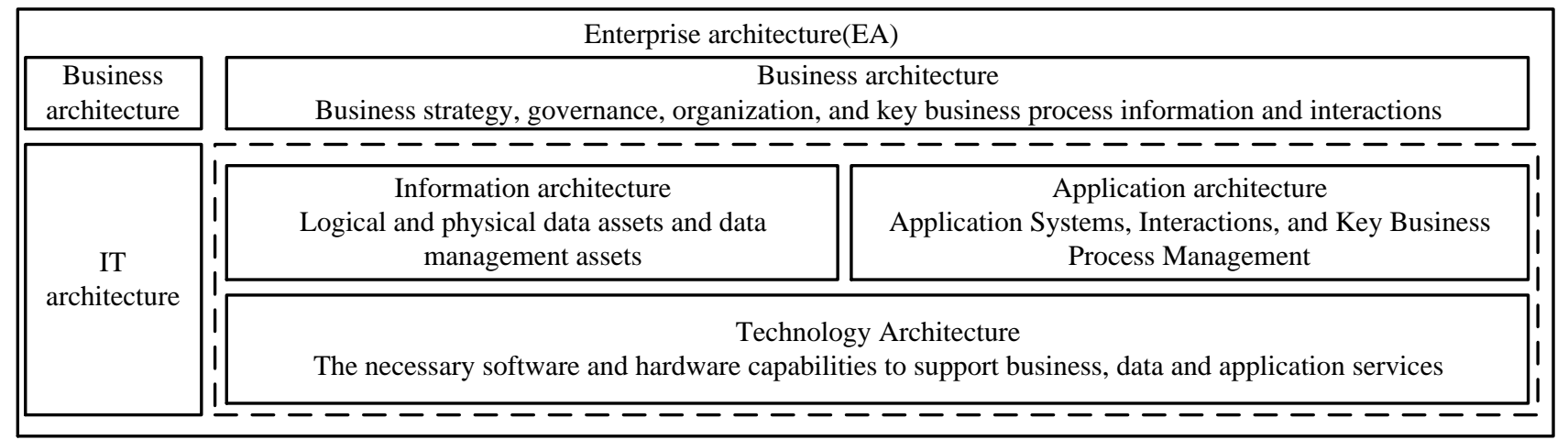

Fig. 1: Enterprise architecture.

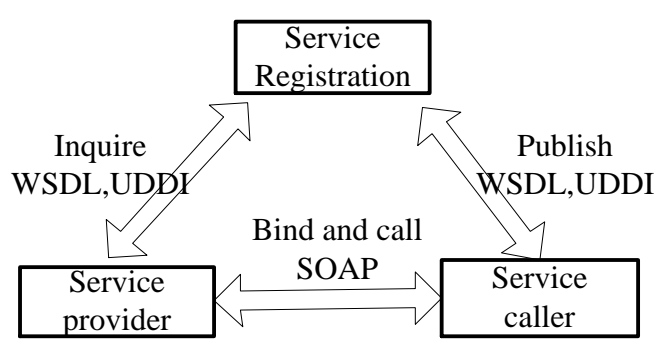

Fig. 2: SOA architecture.

It can be seen that the two architectures generally adopt a hierarchical approach to organize architecture structure (which is shown in Table 1). SOA focuses on the overall architecture of the system and highlights service-oriented aspects. It describes the overall architecture by establishing service models and dividing different levels. However, EA focuses on the system's business architecture, information architecture, application architecture, and technology architecture, weakening the overall architecture of the system. The EA architecture focuses on the macro level, and the SOA architecture focuses on the micro level. Integrating the two architectures to build the overall architecture of the system will bring a qualitative leap forward. After combining EA with SOA, it would be technologically advanced and normative. Through the governance of SOA, it ensures that the architecture meets the requirements of corporate governance in real time, and thus closely cooperates with and penetrates EA in concepts, activities, processes, and structures. SOA would gain the vitality of business after combining EA, and would become the best weapon for SOA landing $^{[2]}$.

Table1: Mapping between SOA and EA architecture field

\begin{tabular}{|l|l|l|}
\hline Architecture & SOA & EA \\
\hline Business & Business Process & Business architecture \\
\hline Application & Serving components & Application architecture \\
\hline Integrated in middleware & Integrated architecture & Technical architecture \\
\hline Data & Data architecture & Information architecture \\
\hline Operating & QoS, Security, Surveillance, and Infrastructure & Technical architecture \\
\hline
\end{tabular}

Considering the fact that multi-professional, multi-service and multi-application in the field of railway construction are interactive and complex, the overall architecture based on the integration of EA and SOA is well adapted to the flexible business, functional complex integration, information fusion sharing, collaborative office work of the railway construction management system. It can effectively solve the information island phenomenon and meet the requirements of system security and scalability.

\section{Integrated Architecture of EA and SOA}




\subsection{The overall Architecture of EA and SOA Integration}

Combining the specific business process and data integration features of the railway construction management system, the overall architecture of the railway construction management system based on EA and SOA integration focuses on SOA and covers the EA's business architecture, application architecture, information architecture, and technology architecture.

The overall structure of railway construction management system is divided as the user access layer, the business application layer, the service composition layer, the data information layer and the infrastructure layer from top to bottom [3]. EA's business, information, application, and technology architecture are integrated in these five layers, and the overall architecture of the railway construction management system that integrates the two is established. (as shown in Figure 3)

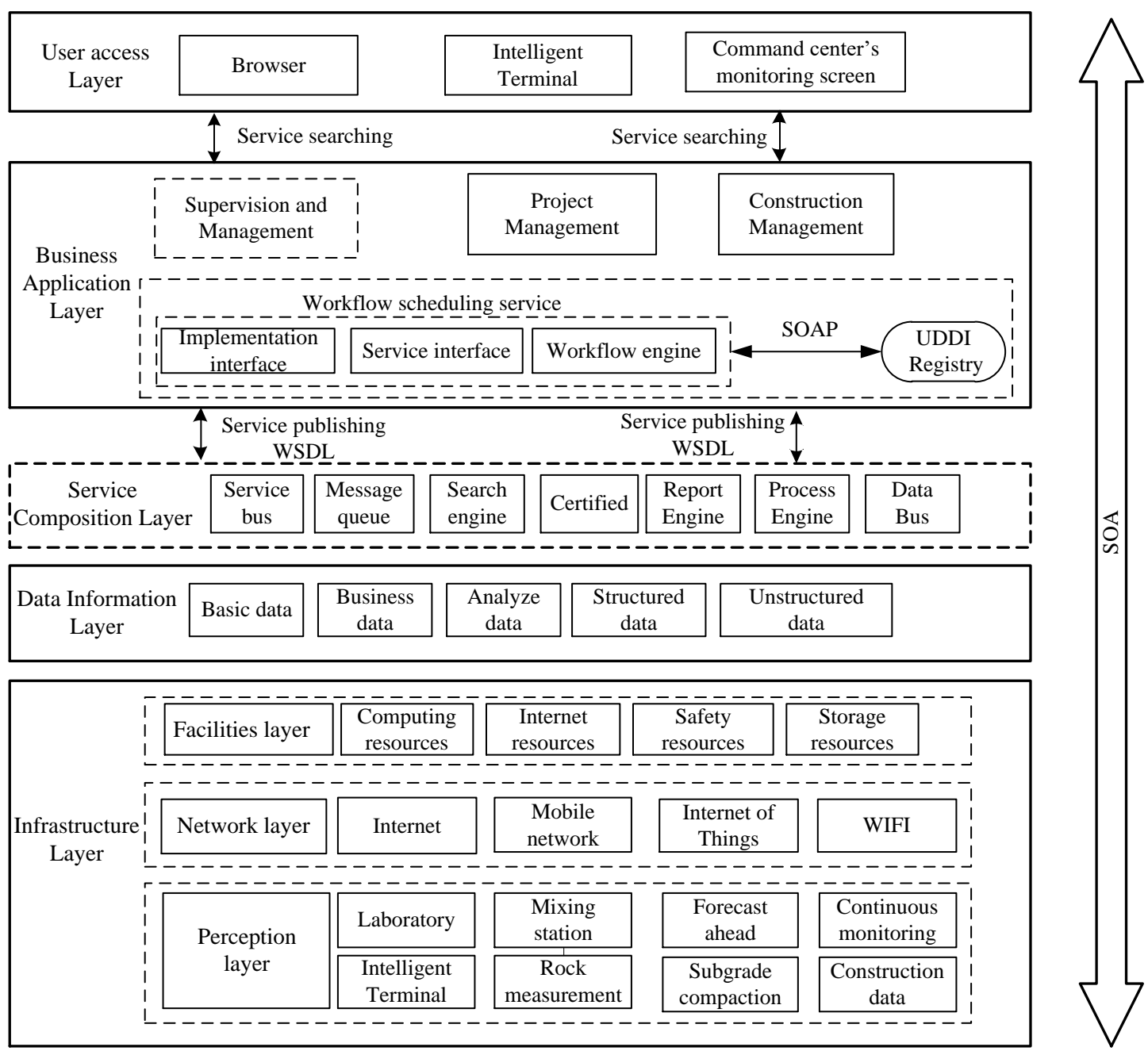

Fig. 3: Overall architecture of EA and SOA convergence.

(1) Infrastructure layer

The infrastructure layer is the infrastructure used to support the operation of the system, including the perception layer, network layer, and facilities layer. The perception layer provides timely and accurate data sources for the system. The network layer transmits the collected data to the data center channel safely and quickly. The facilities layer provides railway construction management system with high performance, high reliability, stable operation, and various effective resources.

This layer embodies the technical architecture model in EA and provides the interaction between various software technologies, hardware technologies, network technologies, and information security technologies to support the normal operation of the entire system.

(2) Data Information Layer 
The data information layer is the service data center that supports the operation of the system. Access to different data is encapsulated into corresponding data services according to requirements. Unified integration on the data service bus is provided for users to call, achieving visual monitoring, standardization, crossplatform access, mass copying for structured data (basic data, business data), and unstructured data (Geographic information, BIM model, document data, and image data).

This layer embodies the information architecture model in EA, which realizes the collection, storage and integration of data resources in railway construction management system, the integration of heterogeneous system data, and the flow of information flow.

(3) Service composition layer

The service composition layer is a collection of system services. The service composition layer comprehensively uses a series of technical services such as enterprise service bus, message queue, authentication and authorization, process engine, and big data processing to complete the composition and arrangement of various heterogeneous information services in the field of railway engineering construction, forming more complicated shared services to meet actual business integration and integration management.

This layer embodies the technical architecture model in EA and implements technology deployment, service composition model, process management model, etc.

(4) Business Application Layer

The business application layer is to interact with the user access layer and is also responsible for invoking service composition layer services. When a user issues a service request through the SOAP protocol, finding and locating the corresponding service in the UDDI service registry through the WSDL file of the web service interfaces in the workflow engine, invoking the service to execute the processing, the result of the execution will be returned to the user for business interaction. By completing the combination and the arrangement of various services through the workflow engine and the enterprise service bus, some more complex services are formed to meet the actual business integration management integration.

This layer embodies the business architecture model in EA, where the transformation from the business objectives of the railway construction management system to an operational business model can be accomplished, describing how the business should be operated to meet the necessary flexibility and extensibility.

(5) User access layer

The user access layer is mainly the SOA architecture request initiating layer. When a user generates requests using some technologies such as SSL VPN, WEB, mobile Internet, being processed through the business layer, service layer, and data layer, the obtained service results will be displayed on the devices such as browser, smart terminal, large screen, which would achieve interactive access to various application service functions of the railway construction management system.

This layer embodies the application architecture model in EA and completes the unified and comprehensive presentation of module information integration of various business functions in the railway construction management system.

Key technologies in this overall architecture are included as follows. An information sharing platform is built based on cloud computing infrastructure and the integration architecture of EA and SOA. Based on OpenStack, KVM, Xen and other open source cloud computing concepts and technical routes, according to user needs, a railway construction management system cloud platform is established to achieve the abilities listed as below.

1) "Pooling" of computing, storage, and network resources.

2) High availability of resources that are on-demand distributed and dynamic extended

3) Data service capabilities of improved information processing and system resource's calculation and storage. 
4) To further protect the security of data information, full-used cloud technology capabilities in resource sharing, disaster recovery, efficiency optimization, systems expansion, operational security, and business integration.

The SOA-based system architecture accommodates the requirements of the four-level management application and meets the requirements for another new project to access the railway construction management system. Through the application of object-oriented EA architecture software development technologies and middleware, the business requirements of flexible configuration can be satisfied.

\subsection{The Business Architecture of EA and SOA Integration}

Through sorting out users of various business activities in railway engineering construction, according to the different work undertaken by different users in the process of railway construction, it is mainly attributed to the three major business areas, namely, construction management, project management and supervision management. Seven types of user organization structure can be summarized, namely, supervision unit, construction unit, survey and design unit, construction unit, regulation unit, evaluation unit, and operation and maintenance unit (as shown in Figure 4).

Key technologies in this overall architecture are included as follows. Based on the business-driven realtime monitoring with early warning and dynamic adjustment technology, the multi-dimensional and multiangle monitored status in "progress, quality, and security" aspects can be displayed in an intuitive and visual way. That is, the railway construction management system monitors the construction site's progress, quality, and safety information in real time. Combined with the organization and management mode of the construction project, it triggers the generation of various warnings and disposal information through the calculation, analysis, and processing of on-site acquisition information, and adopts synchronous technology to issue to various service subsystems to monitor the status of system reception and disposal in real time and support dynamic adjustment of plans and tasks.

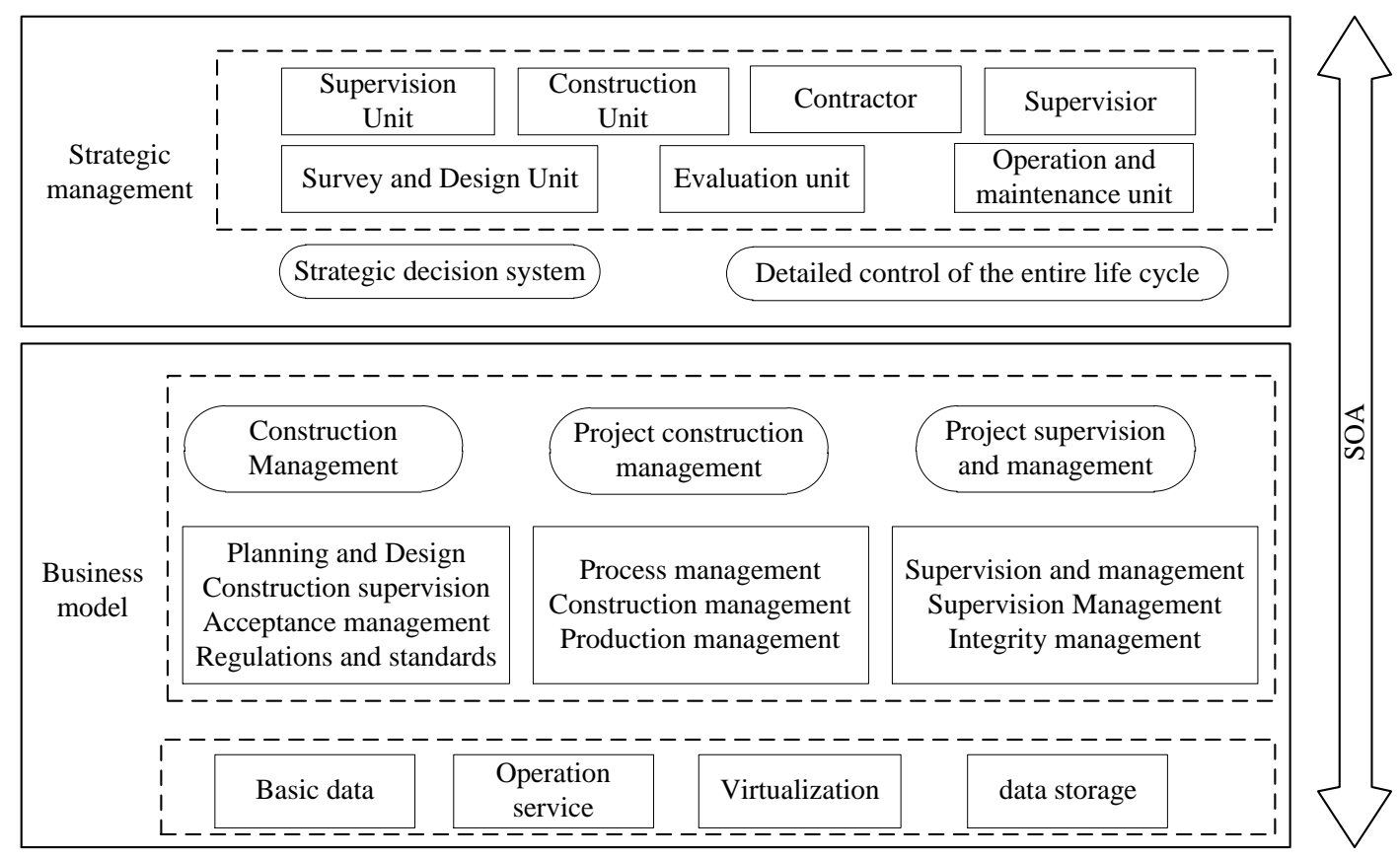

Fig. 4: Converged business architecture based on EA and SOA.

\subsection{EA and SOA Converged Information Architecture}

The information architecture (also called data architecture) of the railway construction management system is based on the theory of EA and abstracts the railway construction business entity into information objects, abstracts the business operation mode into the attributes and methods of the information object, and establishes an object-oriented railway enterprise information model. In order to achieve the transformation 
from the business model to the information model, the mapping of business requirements to information functions, and the abstraction of enterprise basic data to enterprise information $[4,5]$ (as shown in Figure 5)

Key technologies in this overall architecture are included as follows. The information architecture describes the data resources and data flow structure of the railway construction management system from an overall perspective. It mainly revolves around the basic data and business data of the railway construction management system. In accordance with the concept of "classification, definition, management, and integration", the business functions and application data in the system are cleaned, extracted, and converted based on data warehouse technology [6]. Finally, business data is modeled, stored, and delivered. It provides query, analysis, and mining data services for system data centers. At the same time based on WebService, MQ (Message Queue), database sharing and other means of information transmission, to meet the needs of different users to view different projects of business information.

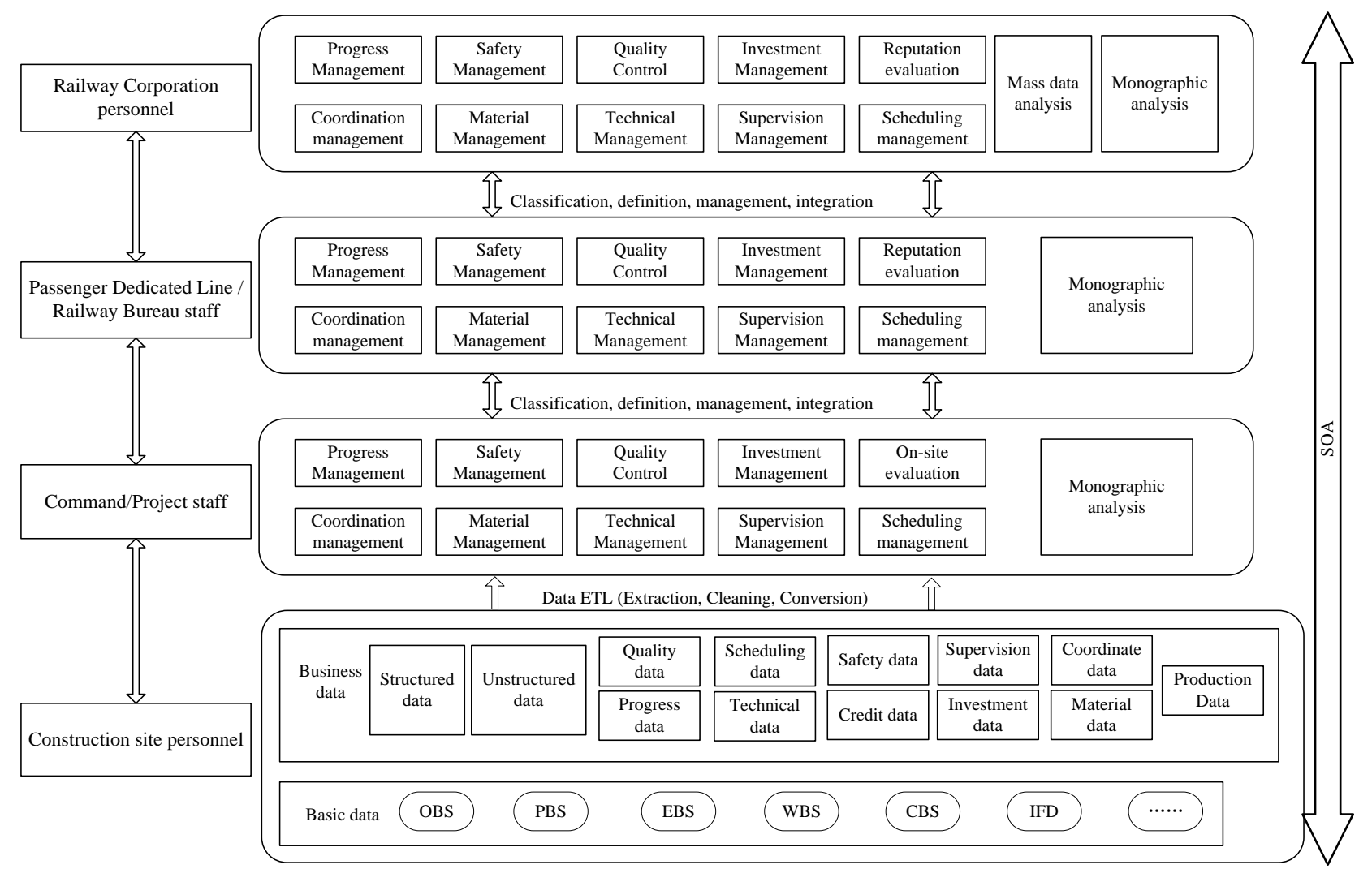

Fig. 5: Converged information architecture based on EA and SOA.

\subsection{EA and SOA Converged Application Architecture}

The application architecture is based on the information architecture and establishes the normal operation of the business functions in the support system. Through the integrated operation of the application system, it increases the interconnection and interoperability of the system, realizes the automatic flow of information, improves the efficiency of the railway construction management business. It reduces the cost and risk of construction management, and provides information, standardization, and digital management support for all professional, full-process, and full-service businesses for railway construction management departments at all levels (as shown in Figure 6).

Key technologies in this overall architecture are included as follows. Basic data management and services based on complex scenarios of railway construction. That is, basic data is the prerequisite for data sharing among various systems and is the core of the construction and management system of railways. The basic data adopts the idea of master data and adopts an object-oriented idea to strictly distinguish the information entities and the relationship between information entities. Some application scenarios use advanced graph databases to meet intricate data relationships. At the same time, it uses big data technology 
to implement data extraction, cleaning, processing, processing, control, and distribution of various basic data [6], and it is formed to meet the needs of various users for different business data applications.

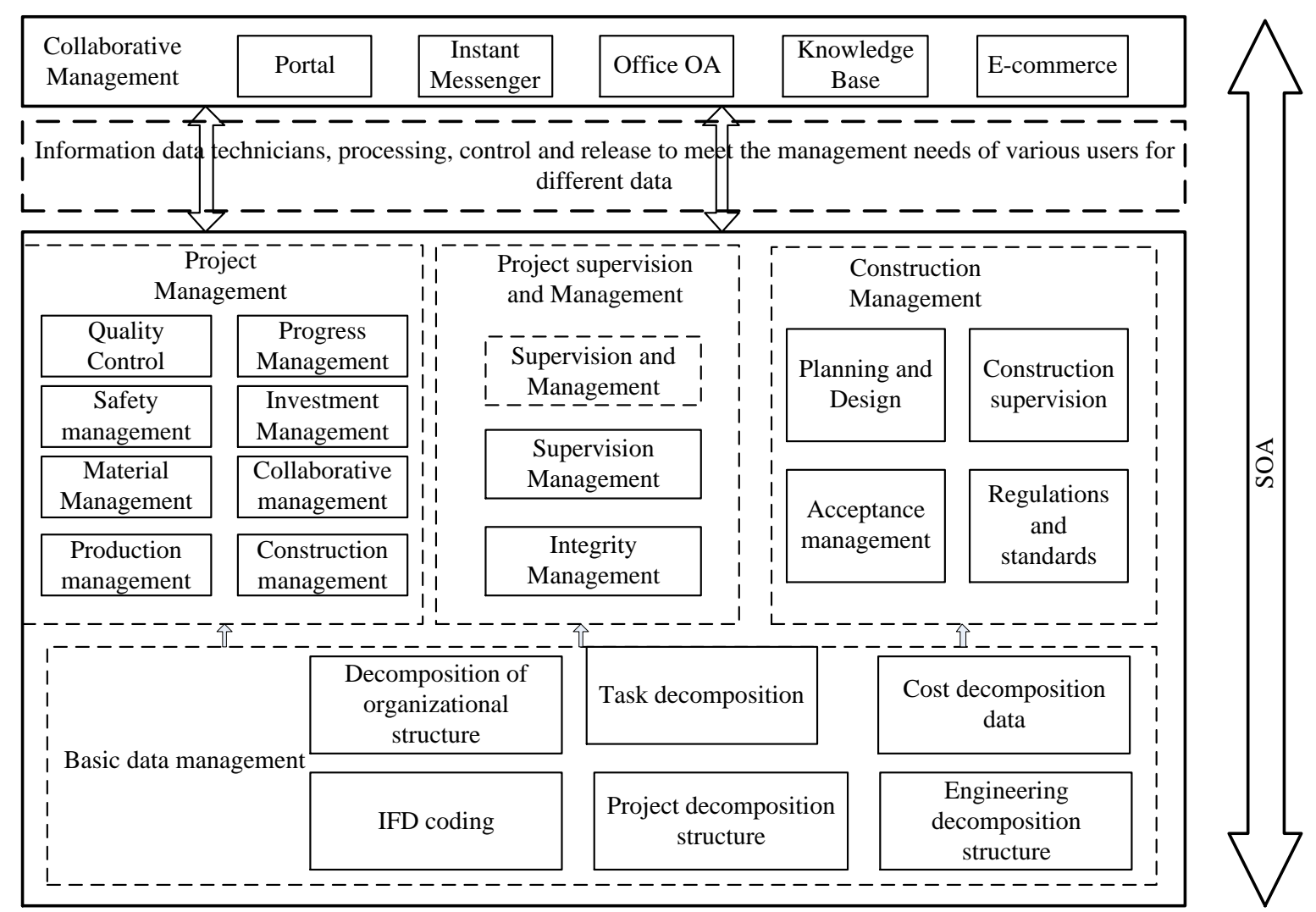

Fig. 6: Converged application architecture based on EA and SOA.

\subsection{EA and SOA Converged Technology Architecture}

The technical architecture is the underlying technology infrastructure for implementing the application architecture. It supports the operation of system applications through interactions between software, hardware, networks, and information security technologies. It is the technical implementation of the entire system, and the deployment, distribution, and technology environment of the system. From the perspective of technology implementation, the technical structure of the railway construction management system is divided into five layers (as shown in Figure 7).

Key technologies in this overall architecture are included as follows. Railway construction based on mobile internet is extensive in IntelliSense system. Using the perception technology of the internet to build a space-time self-inductance system for railway engineering construction. Through real-time perception and collection of various types of data, build three-level perception networks of people and people, people and things, and people and nature, and achieve information accessibility and transmission. Achieve comprehensive awareness of the construction site's natural conditions, operating personnel, project entities, equipment supplies, and other engineering elements, and achieve an overall barrier-free transmission of construction sites and participating parties to form a three-dimensional mobile transmission system.

\section{System Network Architecture}

The railway construction management system adopts a four-level networking scheme and is divided into four levels: the railway company, the railway bureau/customer company, the target segment and the project site. The overall system is mainly divided into three types of applications: Intranet applications, Internet applications, and mobile applications.

Key technologies in this overall architecture are included as follows. The railway construction management system is deployed in the cloud service center based on the Internet. Security measures such as 
WAF, firewall, security platform, and VPN are used to improve the security and reliability of data transmission. Secure access to the system through security access control, security agents, and dynamic physical isolation mechanisms. Use special planes for users of construction units to access intranet OA systems through VPN security mechanisms. Sensitive data applications on the mobile end, 24/7 security protection of mobile devices through MDM security protection. Internet users use the security platform device to isolate the Internet zone from the internal private network zone to effectively prevent Internet users from attacking the intranet and protect the intranet server. (as shown in Figure 8).

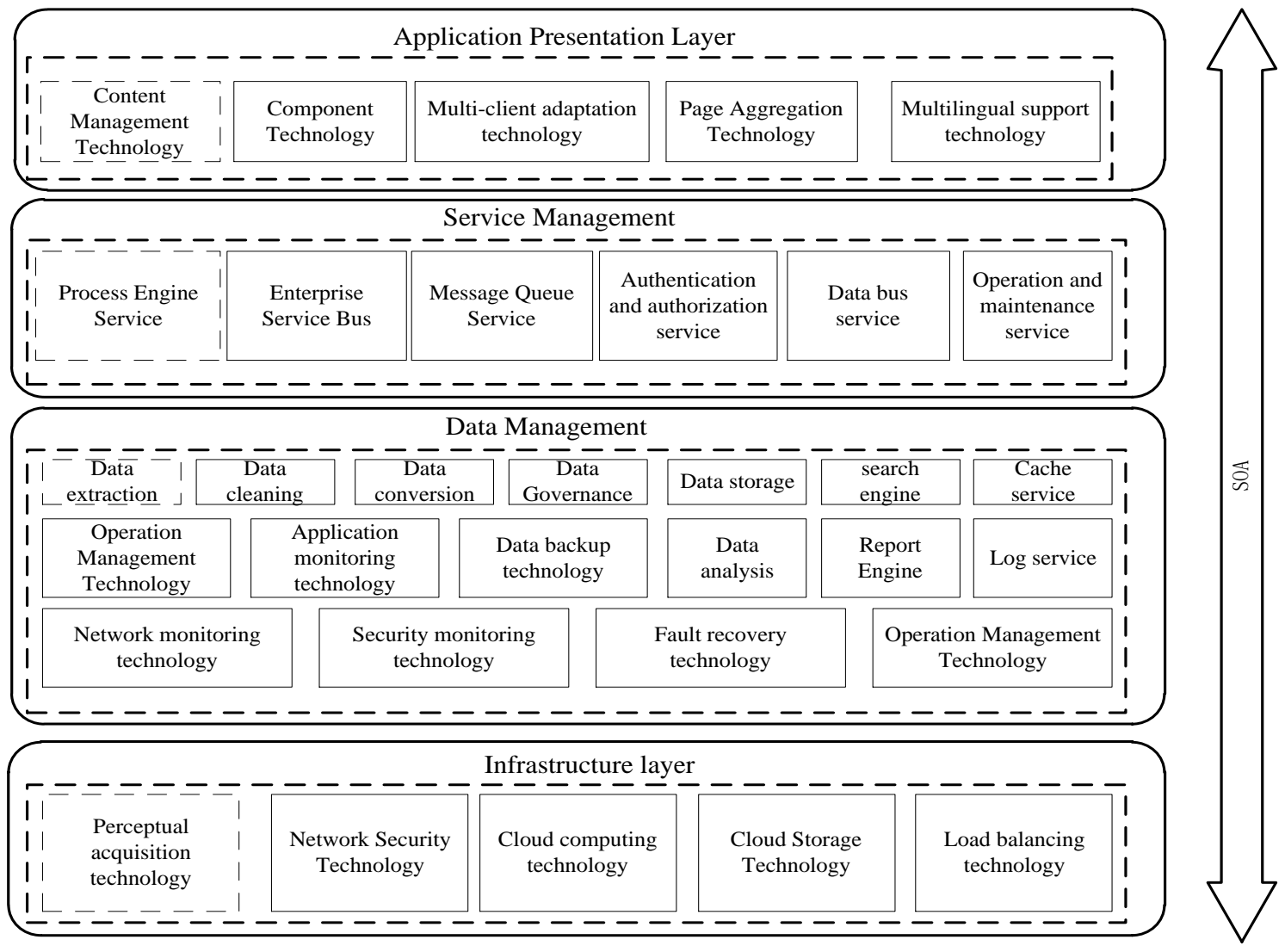

Fig. 7: Converged technology architecture based on EA and SOA.

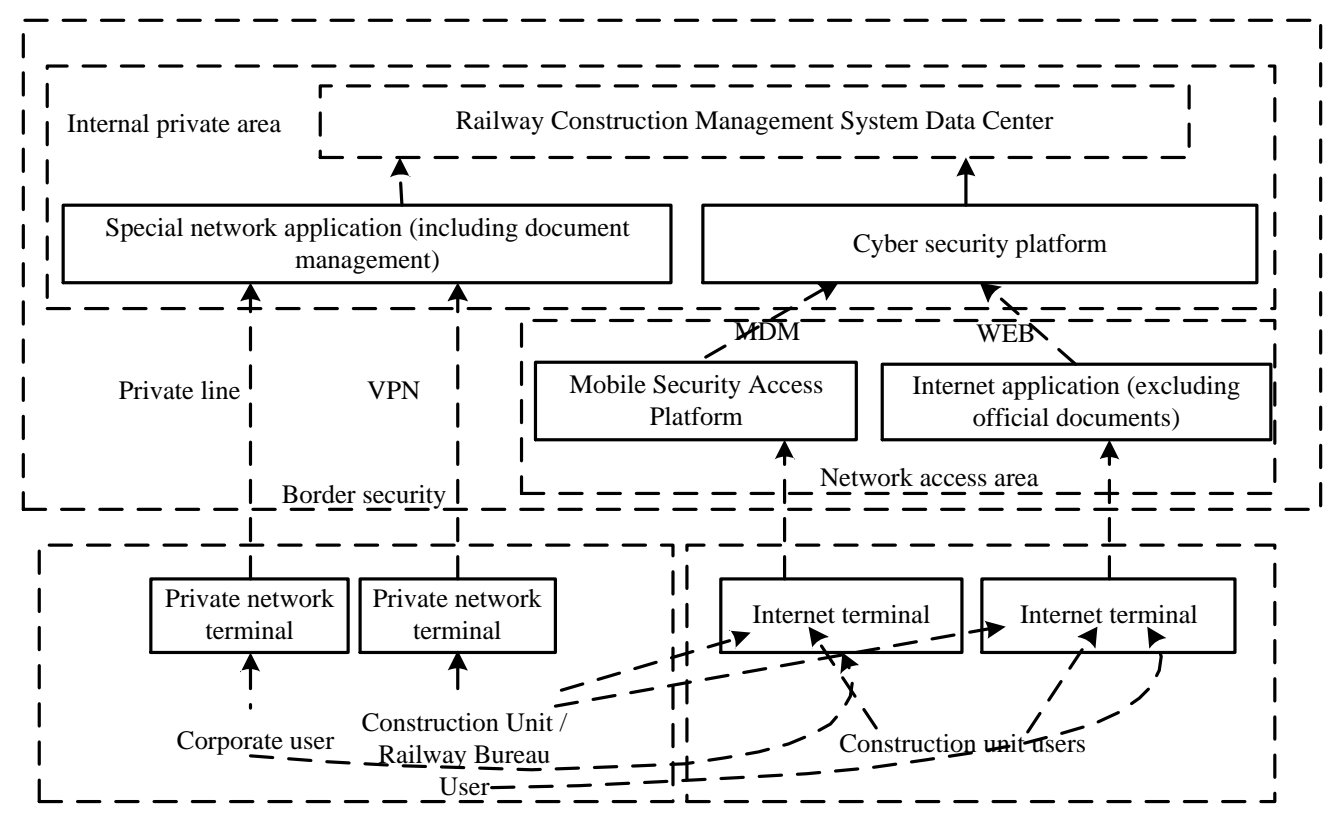

Fig. 8: System network diagram. 


\section{System Security Solution}

Under the guidance of the company's information security specification and the second level of the information system security protection level, through the physical security, network security, host security, data security, application security and security management platform (shown in Figure 9), to build railway construction management Information security protection system.

Key technologies in this overall architecture are included as follows. Physical safety provides a safe and reliable operating environment to ensure stable and reliable operation. According to the importance of different domains, adopt different levels of security policies to achieve network security protection. Host security guarantees the security of application cloud systems and data center host systems. Data security enables data availability, data confidentiality and integrity. Application security adopts identity authentication and access control, digital signature, software fault tolerance, resource control, mobile security application security. Security management platform realizes unified and centralized security protection management of data centers.

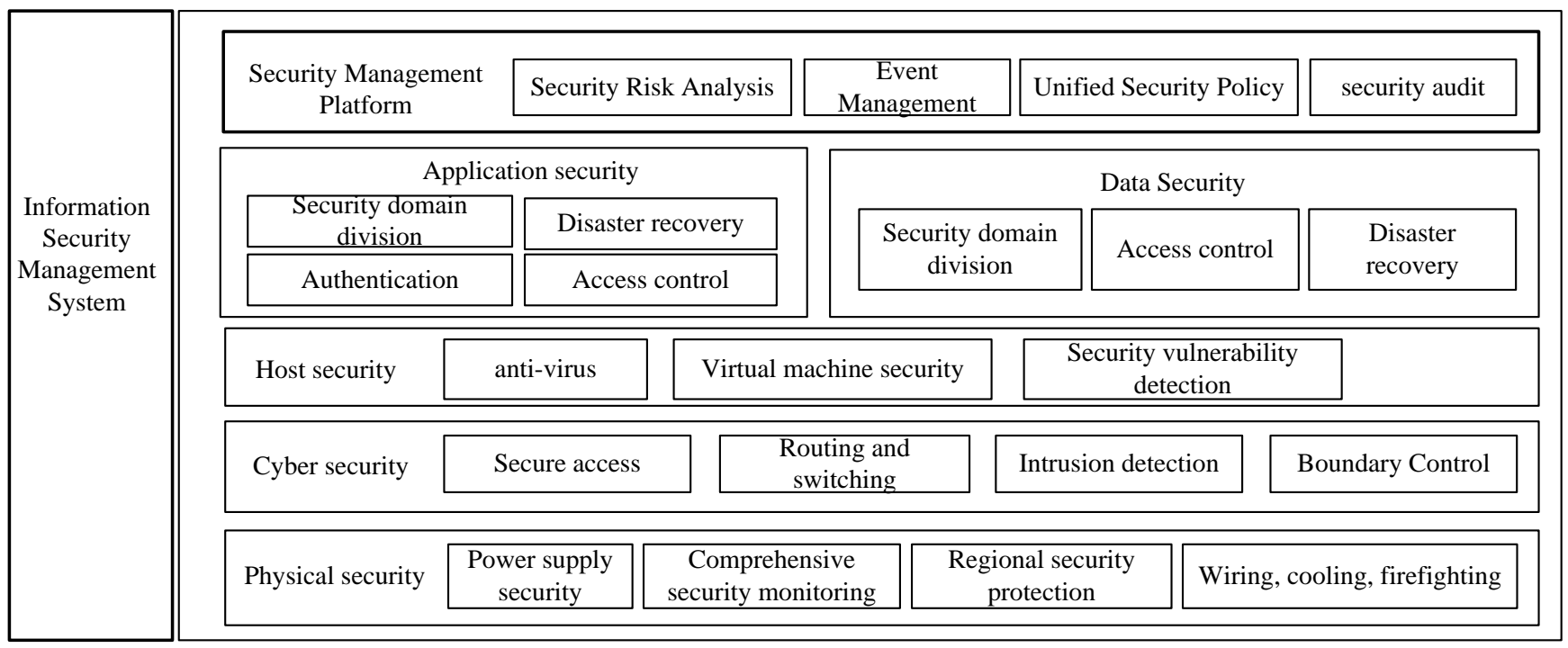

Fig. 9: System security protection system.

\section{Conclusion}

In this paper, combining with the multi-professional, multi-service and multi-application interactions in the railway construction field, based on the EA and SOA integration technology abstracting the railway construction business model, the overall technical solution for the railway construction management system based on SOA and EA integration is proposed. Various services or applications and services can be mutually accessible. It greatly optimizes the system connection architecture, reduces the complexity of system integration, and effectively avoids the problems of high system integration complexity, poor scalability, and system fragility in traditional systems.

The system has been officially put into use during the railway construction project. Combining with the actual engineering application verification, it solves the technical application problems of the original system's collaborative sharing and flexible business change. Satisfy the actual needs of the railway construction business management, achieved good application results, ensure the safety and quality of the project, improve the efficiency of collaborative operations and reduce the cost of project construction.

\section{Future Works}

Although the EA and SOA based railway construction management system's data combination, information sharing, and the integrated application of key businesses have been achieved, the depth of information combination and the range of business application need to be further improved and perfected. If 
further combined with the grid technology and the cloud computing to study and improve the underlying infrastructure, the resource sharing, data fusion, and a huge amount of application integration of the railway construction management system could be achieved in a real sense, which can contribute to the decrease of a waste of duplicated information system construction resource and the construction cost reduction.

\section{Acknowledgements}

We gratefully thank the anonymous reviews for their valuable feedback. This research was supported by the Science and Technology Research and Development Key Program of China Railway Corporation (CRC) under grants No. 2016X002, No. 2017G001-B, No. 2017G001-D, and No. 2017G001-E. This research was also supported by the Science and Research Major Program of China Railway Research Institute (CRRI) under grants No. 2016YJ098. Any opinions, findings, and conclusions expressed in this material are those of the authors and do not necessarily reflect the views of the CRC and CRRI.

\section{References}

[1] Rosen M, Lublinsky B, Smith K T, et al. Applied SOA: service-oriented architecture and design strategies [M]. John Wiley \& Sons, 2012.

[2] YANG Guoyuan, SHI Tianyun, FANG Kai, ZHANG Qiuliang. General framework and key technologies of Railway Passenger Transport Management Information System[J]. Railway Computer Application, 2016, 25(3): 22-26.

[3] SHI Xiang, ZHANG Guodong, SUNWeihong. SOA oriented Information Architecture Planning for Gas Enterprises[J]. Computer Engineering and Design, 2012, 33(4): 1418-1423.

[4] LAI Maosheng,WANG Jing, ZHANG Mengdi. An Innovative Study the Government Information Resources Management and Enterprise Architecture Theory[J]. Library and Information Service, 2014, 58(6):12-18.

[5] GUO Lusheng, LIU Chunnian. Research on Government Emergency Information Resource Planning Based on EA under Big Data Environment[J]. Journal of Intelligence, 2016, 35(6):171-176.

[6] CAO Jianjun, DIAO Xingchun, CHEN Shuang. Data Cleaning and its General System Framework[J], Computer Science, 2012, 39(11A): 207-211. 